\title{
Synthetic logic
}

\author{
Alex J. Djalali ${ }^{1}$
}

\begin{abstract}
The role of inference as it relates to natural language (NL) semantics has often been neglected. Recently, there has been a move away by some NL semanticists from the heavy machinery of, say, Montagovianstyle semantics to a more proof-based approach.

Although researchers tend to study each type of system independently, MacCartney (2009) and MacCartney and Manning (2009) (henceforth $\mathrm{M} \& \mathrm{M})$ recently developed an algorithmic approach to natural logic that attempts to combine insights from both monotonicity calculi and various syllogistic fragments to derive compositionally the relation between two NL sentences from the relations of their parts.

At the heart of their system, M\&M begin with seven intuitive lexicalsemantic relations that NL expressions can stand in, e.g., synonymy and antonymy, and then ask the question: if $\varphi$ stands in some lexicalsemantic relation to $\psi$; and $\psi$ stands in (a possibly different) lexicalsemantic relation to $\theta$; what lexical-semantic relation (if any) can be concluded about the relation between $\varphi$ and $\theta$ ? This type of reasoning has the familiar shape of a logical inference rule.

However, the logical properties of their join table have not been explored in any real detail. The purpose of this paper is to give M\&M's table a proper logical treatment. As I will show, the table has the underlying form of a syllogistic fragment and relies on a sort of generalized transitive reasoning.
\end{abstract}

\section{Introduction}

The role of inference as it relates to natural language (NL) semantics has oft been neglected. Recently, there has been a move away by some NL semanticists from the heavy machinery of, say, Montagovian-style semantics to a more proof-based approach. This represents a belief that the notion of derivability plays as central a role in NL semantics

\footnotetext{
${ }^{1}$ Stanford University

LiLT Volume 9

Perspectives on Semantic Representations for Textual Inference. Copyright (C) 2014, CSLI Publications.
} 
as that of entailment. Beginning with van Benthem (1986), and continuing on with Valencia (1991), Dowty (1994), Gilad and Francez (2005), Moss (2008), Moss (2010), van Benthem (2008), Moss (2009) and Moss (2012) among others, the study of various natural logics has become commonplace.

Natural logicians place an emphasis on the development and study of proof theories that capture the sort of inferences speakers of a particular NL like English make in practice. It should be said, though, that 'natural logic' is a catchall term that refers to either the study of various monotonicity calculi à la van Benthem or Aristotelean-style syllogistic fragments à la Moss. Although researchers tend to study each type of system independently, MacCartney (2009) and MacCartney and Manning (2009) (henceforth M\&M) recently developed an algorithmic approach to natural logic that attempts to combine insights from both monotonicity calculi and various syllogistic fragments to derive compositionally the relation between two NL sentences from the relations of their parts.

At the heart of their system, M\&M begin with seven intuitive lexicalsemantic relations that NL expressions can stand in, e.g., synonymy and antonymy, and then ask the question: if $\varphi$ stands in some lexicalsemantic relation to $\psi$; and $\psi$ stands in (a possibly different) lexicalsemantic relation to $\vartheta$; what lexical-semantic relation (if any) can be concluded about the relation between $\varphi$ and $\vartheta$ ? This type of reasoning has the familiar shape of a logical inference rule, a schema of which is given in (1):

$$
\frac{\varphi R \psi \quad \psi S \vartheta}{\varphi T \vartheta}
$$

Drawing from their stock of lexical-semantic relations, for every instance of $R$ and $S$, M\&M reason semantically to calculate $T$, and present their results in what they call a join table. However, to my knowledge at least, the logical properties of their join table have not been explored in any real detail. The purpose of this paper is to give M\&M's table a proper logical treatment. As I will show, the table has the underlying form of a syllogistic fragment and relies on a sort of generalized transitive reasoning. Here, I define a basic set-theoretic semantics and proof calculus for M\&M's join table and prove a completeness theorem for it.

\section{Synthetic Logic}

I begin first by defining the syntax of a synthetic language $\mathcal{S Y N}$.

Definition 2.1 (Syntax of $\mathcal{S} \mathcal{Y N}$ ). Let $p_{1}, \ldots p_{n}$ be atoms for $n<\omega$, 
which themselves are all elements of $\Phi$ the set of proper terms. Then

1. If $\varphi$ is a proper term, then so is $\bar{\varphi}$. Nothing else is a proper term.

2. If $\varphi$ and $\psi$ are proper terms, then

$$
\begin{aligned}
& \varphi \equiv \psi, \quad \varphi \sqsubset \psi, \quad \varphi \sqsupset \psi, \\
& \varphi^{\wedge} \psi, \quad \varphi \rrbracket \psi, \quad \varphi \smile \psi
\end{aligned}
$$

are synthetic terms.

I let $\mathcal{S Y \mathcal { N }}$ be the smallest set containing both sets of proper and synthetic terms given by definition 2.1. I say $\bar{\varphi}$ is the complement of $\varphi$ and refer to the set $\mathcal{M}=\{\equiv, \sqsubset, \sqsupset, \wedge, \mathbb{N}, \smile\}$ as the set of MaCcartney relations, as they are taken from MacCartney (2009) and MacCartney and Manning (2009). The relations themselves can be read as equality, strict forward and reverse entailment, negation, alternation and cover respectively. I use $R, S$ and $T$ as meta-logical variables ranging over elements of $\mathcal{M}$; and I will use $\varphi, \psi$ and $\vartheta$ as meta-logical variables ranging over proper terms. Finally, I assume that $\varphi \equiv \overline{\bar{\varphi}}$ for all $\varphi$.

In definition 2.1, I use the term 'atoms' as opposed to, say, 'proposition letters'. This is purposeful. Intuitively, there is nothing that prevents the atoms of a synthetic language $\mathcal{S Y \mathcal { N }}$ from being NL expressions of any type, assuming some sort of syntactic typing. From a formal perspective, this would simply amount to considering a family of synthetic languages $\left\{\mathcal{S Y N}_{\alpha} \mid \alpha \in\right.$ Types $\}$, where Types is the set of, say, Montagovian types. To better understand this, suppose

$$
\text { run } \sqsubset \text { move }
$$


itively, (2) makes the meta-semantic statement, "The intransitive verb run strictly forward entails the intransitive verb move". In this way, synthetic languages are quite general - they just are languages of the lexicon.

No doubt synthetic languages are impoverished, as they lack the classical boolean connectives. This is not to say that a synthetic language could not be extended. I refer the reader to Moss (2010) for a natural logic with boolean connectives both inside and out. In our setting, the addition of 'inside' boolean connectives would be to augment the class of proper terms by, say, the elements of the set $\{\neg, \wedge\}$. This would result in terms like the following:

$$
\varphi \wedge \psi
$$

where $\varphi$ and $\psi$ are proper terms.

To have 'outside' boolean connectives would be to augment the class of synthetic terms by a functionally complete set of connectives like the 
one above. Examples of such terms would be

$$
(\varphi \sqsubset \psi) \wedge(\vartheta \sqsupset \chi)
$$

where $\varphi, \psi, \vartheta$ and $\chi$ are proper terms. Given both outside and inside boolean connectives, the following would be a valid expression exhibiting both types of coordination:

$$
((\varphi \wedge \psi) \sqsubset \varphi) \vee((\varphi \wedge \psi) \sqsupset \varphi)
$$

Without relying on semantic intuitions, it may be difficult to intuit the difference between inside and outside Boolean connectives. From a syntactic perspective, the analogy, here, is between entity level, or more generally, non-sentential level, coordination in a NL language like English, e.g., John and Mary, versus sentential level coordination, e.g., John went to the store and Mary went to the store. The former would be an instance of inside Boolean coordination, and the latter would be an instance of outside Booelean coordination. As it turns out, augmenting definition 2.1 in this way would be productive for reasons which will become clearer. However, I leave this extension for future work.

Turning now to the semantics of $\mathcal{S Y \mathcal { N }}$, I begin first by defining the sorts of models I will be working with.

Definition 2.2 (Synthetic Models). Let a synthetic model $\mathbb{M}$ be the pair $\langle D, A A A \cdot B B B\rangle$, where $D$ is a non-empty set and $A A A \cdot B B B$ is an interpretation function such that $A A A \varphi B B B \subseteq D$ and

1. $A A A \bar{\varphi} B B B=D-A A A \varphi B B B$;

2. $A A A \overline{\bar{\varphi}} B B B=A A A \varphi B B B$;

3. $A A A \varphi B B B \neq A A A \bar{\varphi} B B B$; and

4. $A A A \varphi B B B \neq\left\{\begin{array}{l}\varnothing \\ D\end{array}\right.$ or

for all proper terms $\varphi$

The first three conditions on the interpretation function $A A A \cdot B B B$ force the standard semantics of set-theoretic complementation. The fourth condition restricts the possible model space to just those models which interpret each proper term non-vacuously, where a 'non-vacuous' term is a term which denotes neither the domain in its entirety nor the empty-set. In fact, I call the logic 'synthetic' after Popper (1968) who argues that "synthetic statements in general are placed, by the entailment relation, in the open interval between self-contradiction and tautology".2

\footnotetext{
${ }^{2}$ Instead of reanalyzing non-vacuous terms, such as the square circle as a pred-
} 


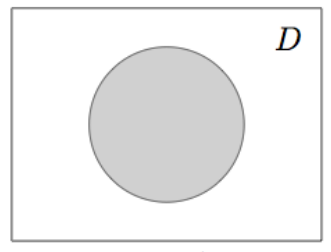

$\varphi \equiv \psi$
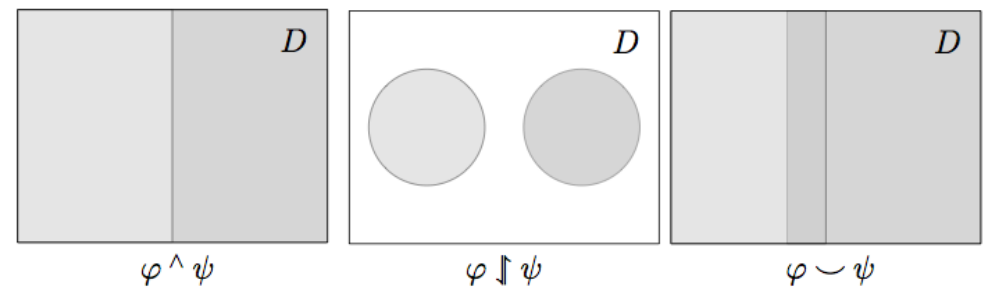

FIGURE 1: A graphical representation of the $\mathcal{M}$-relations

The semantics of the various synthetic terms can be naturally defined set-theoretically, as in definition 2.3. A pictorial representation of the semantics of each relation is shown in figure 1.

Definition 2.3 (Tarski-Style Truth-Conditions). Let $\varphi$ and $\psi$ be proper terms and $R$ a $\mathcal{M}$-relation. Define the denotation of the synthetic term $\varphi R \psi$, written $A A A \varphi R \psi B B B$, as follows:

$$
\begin{aligned}
& \mathbb{M}=\varphi \equiv \psi \Leftrightarrow A A A \varphi B B B=A A A \psi B B B \\
& \mathbb{M}=\varphi \sqsubset \psi \Leftrightarrow A A A \varphi B B B \subset A A A \psi B B B \\
& \mathbb{M}=\varphi \sqsupset \psi \Leftrightarrow A A A \varphi B B B \supset A A A \psi B B B \\
& \mathbb{M}=\varphi^{\wedge} \psi \Leftrightarrow(A A A \varphi B B B \cap A A A \psi B B B=\varnothing) \wedge(A A A \varphi B B B \cup \\
& A A A \psi B B B=D) \\
& \mathbb{M}=\varphi \llbracket \psi \Leftrightarrow(A A A \varphi B B B \cap A A A \psi B B B=\varnothing) \wedge(A A A \varphi B B B \cup \\
& A A A \psi B B B \neq D) \\
& \mathbb{M}=\varphi \smile \psi \Leftarrow(A A A \varphi B B B \cap A A A \psi B B B \neq \varnothing) \wedge(A A A \varphi B B B \cup \\
& A A A \psi B B B=D)
\end{aligned}
$$

icate having an empty-extension à la Russell (1905), M\&M bar them from their logic altogether, claiming that such terms fail to divide the world into meaningful conceptual categories. This might seem odd to logicians or philosophers, however, as M\&M's formal system is the basis for this work, I see no harm in assuming it here. 


\begin{tabular}{|c|cccccc|}
\hline$R, S$ & $\equiv$ & $\sqsubset$ & $\sqsupset$ & $\wedge$ & $\mathbb{}$ & $\smile$ \\
\hline$\equiv$ & $\equiv$ & $\sqsubset$ & $\sqsupset$ & $\wedge$ & $\mathbb{}$ & $\smile$ \\
$\sqsubset$ & $\sqsubset$ & $\sqsubset$ & $\cdot$ & $\mathbb{}$ & $\mathbb{}$ & $\cdot$ \\
$\sqsupset$ & $\sqsupset$ & $\cdot$ & $\sqsupset$ & $\smile$ & $\cdot$ & $\smile$ \\
$\wedge$ & $\wedge$ & $\smile$ & $\mathbb{}$ & $\equiv$ & $\sqsupset$ & $\sqsubset$ \\
$\downarrow$ & $\Downarrow$ & $\cdot$ & $\Downarrow$ & $\sqsubset$ & $\cdot$ & $\sqsubset$ \\
$\smile$ & $\smile$ & $\smile$ & $\cdot$ & $\sqsupset$ & $\sqsupset$ & $\cdot$ \\
\hline
\end{tabular}

FIGURE 2: $\mathcal{M}$-Rules

No doubt the logic could be simplified drastically by giving the semantics of forward entailment in terms of (set-theoretic) sloppy forward containment $(\subseteq)$ and negation $(\neg)$ and defining the other $\mathcal{M}$-relations in terms of these connectives. However, M\&M make the purposeful decision to effectively tease apart the $\subseteq$ relation into two different ones. I follow suit and sacrifice formal elegance in attempt to capture a finite, primitive stock of semantic relations that humans, at least intuitively, seem to reason in terms of. Having said that, the meta-logical relation of entailment will be defined in the usual way.

Definition 2.4 (Entailment). Let $\Gamma$ be a set of synthetic terms. $\Gamma$ entails $\varphi R \psi$ written, $\Gamma \models \varphi R \psi$ just in case

$$
\mathbb{M}=\varphi^{\prime} S \psi^{\prime} \Rightarrow \mathbb{M}=\varphi R \psi
$$

for all $\varphi^{\prime} S \psi^{\prime} \in \Gamma$.

To conclude this section, the members of $\mathcal{M}$ can be shown to be mutually exclusive.

Theorem 2.1 (Mutual Exclusivity of the $\mathcal{M}$-relations). If $\mathbb{M}$ is a synthetic model then

for $R \neq S$.

$$
\mathbb{M}=\varphi R \psi \Rightarrow \mathbb{M} \not \models \varphi S \psi
$$

The proof is trivial but tedious and relies on basic set theory and the fact that all proper terms are interpreted non-vacuously.

\subsection{The Proof Calculus}

The proof system of this logic effectively takes the form of a natural deduction system. There will be two types of rules: $M$-rules and $D$ rules, the former named after MacCartney because they are effectively the rules he works with in his dissertation; and the latter so-named to distinguish the fact that I have added them in this paper to ultimately 
create a complete proof-calculus.

I begin with the former, a schema of which is given in definition 2.5.

Definition 2.5 ( $M$-Rules). Let $\Gamma$ be a set of synthetic formulas. Then,

$$
\frac{\Gamma \vdash \varphi R \psi \quad \Gamma \vdash \psi S \vartheta}{\Gamma \vdash \varphi T \vartheta} R, S
$$

are rules of the calculus.

This schema is instantiated by taking $T$ to be the $\mathcal{M}$-relation gotten by intersecting the $\mathcal{M}$-relations in the $R$-row and $S$-column in Figure (2). So, for example, (6) is a valid rule in the synthetic proof calculus:

$$
\frac{\Gamma \vdash \varphi \equiv \psi \quad \Gamma \vdash \psi \sqsubset \vartheta}{\Gamma \vdash \varphi \sqsubset \vartheta} \equiv, \sqsubset
$$

It reads, 'If there are derivations from a set of premises $\Gamma$ of $\varphi \equiv \psi$ and $\psi \sqsubset \vartheta$, then there is a derivation of $\varphi \sqsubset \vartheta$ from the same set of premises'.

Figure 2 is a modified version of M\&M's join table. In their table, the value of the cells marked here with the dot '.' correspond to a disjunction of synthetic terms. Translating these cells of their table in terms of the calculus presented here, the pseudo-rule $\sqsubset, \sqsupset$ would be given as follows:

$$
\frac{\Gamma \vdash \varphi \sqsubset \psi \quad \Gamma \vdash \psi \sqsupset \vartheta}{\Gamma \vdash(\varphi \equiv \vartheta) \vee(\varphi \sqsubset \vartheta) \vee(\varphi \sqsupset \vartheta) \vee(\varphi \rrbracket \vartheta)} \sqsubset, \sqsupset
$$

Although M\&M do not define a proper proof a calculus, in this instance, their semantic reasoning reasoning would be as follows:

- Given that $(i)$ the set denoted by $\varphi$ is strictly contained in the set denoted by $\psi$, and (ii) the set denoted by $\vartheta$ is strictly contained in the set denoted by $\psi$, what can one say about the set-theoretic relation that holds between the sets denoted by $\varphi$ and $\chi$ ?

- One can certainly construct a model in which the set denoted by $\varphi$ is equal to the set denoted by $\vartheta$; but one can construct a model in which the set denoted by $\varphi$ is strictly contained in the set denoted by $\vartheta$; but one can also construct ... and so on and so forth for all the disjuncts in (7).

- Given $(i)$ and $(i i)$, at best, one can say that $\varphi$ is equivalent to $\vartheta$ or $\varphi$ strictly forward entails $\vartheta$ or ... and so on and so forth. ${ }^{3}$

\footnotetext{
${ }^{3} \mathrm{M} \& \mathrm{M}$ observe that, given the assumptions in $(i)$ and $(i i)$, it is not possible to construct a model in which $\varphi$ and $\vartheta$ denote complementary sets, lest a contradiction ensue. So, they do not include the synthetic statement $\varphi^{\wedge} \vartheta$ in the disjunctive statement. (Similarly, for $\varphi \smile \vartheta$ ).
} 
The logic, as I have laid it out here, is not expressive enough to capture the above disjunctive reasoning. This is because it lacks 'outside' boolean connectives in the sense made explicit above. This being the case, I omit the values in figure 2 marked with a ' ' from being possible instantiations of the above schema.

Although not in M\&M's original system, I include the following proof rules in the calculus:

Definition 2.6 ( $D$-rules). Again, let $\Gamma$ be a set of synthetic terms. Then,

$$
\begin{array}{llll}
\frac{\Gamma \vdash \varphi \equiv \psi}{\Gamma \vdash \varphi \equiv \varphi} \equiv_{1} & \frac{\Gamma \vdash \psi}{\Gamma \vdash \psi \equiv \varphi} \equiv_{2} & \frac{\Gamma \vdash \varphi^{\wedge} \psi}{\Gamma \vdash \bar{\varphi}} \wedge_{1} & \frac{\Gamma \vdash \varphi_{2}}{\Gamma \vdash \psi^{\wedge} \varphi}{ }_{2} \\
\frac{\Gamma \vdash \varphi \sqsubset \psi}{\Gamma \vdash \psi \sqsupset \varphi} \sqsubset_{1} & \frac{\Gamma \vdash \varphi \sqsupset \psi}{\Gamma \vdash \psi \sqsubset \varphi} \sqsupset_{1} & \frac{\Gamma \vdash \varphi \rrbracket \psi}{\Gamma \vdash \psi \sqrt{ }{ }_{1}} & \frac{\Gamma \vdash \varphi \smile \psi}{\Gamma \vdash \psi \smile \varphi} \smile_{1} \\
\frac{\varphi \in \Gamma}{\Gamma \vdash \varphi} \text { Refl } &
\end{array}
$$

are rules of the calculus.

The $D$-rules syntactically encode for the basic relational properties of the $\mathcal{M}$-relations. $\equiv_{2}$, for example, allows us to prove the fact that settheoretic equality is symmetric.

From a logical perspective, $\Gamma$ is nothing more than a set of premises. However, in natural logic, it is understood as being a lexicon that encodes for the basic lexical semantic relations expressions of a language stand in. A sample lexicon might look something like the following:

$$
\Gamma=\left\{\text { Dutchman } \int \text { Frenchman, Dutchman } \sqsubset \operatorname{man}\right\}
$$

In (8), $\Gamma$ encodes for the fact that the noun Dutchman alternates with the noun Frenchman and the fact that the noun Dutchman strictly forward entails the noun man. (In this case, I am working with a synthetic language of common nouns).

More generally, from a lexicon, various other relations that natural language statements stand in can be proven. To see how the synthetic proof calculus works in practice, consider the following derivations shown in theorem 2.2:

Theorem 2.2. Let $\Gamma$ be a set of premises. The following theorems are all derivable in the proof calculus: ${ }^{4}$

\footnotetext{
${ }^{4}$ I also provide natural language instances of each theorem to demonstrate that given certain intuitive assumptions about the relations natural language expressions stand in, other intuitive statements about those relations can be inferred.
} 
1. $\Gamma^{\prime}, \varphi \equiv \psi \vdash \varphi^{\wedge} \bar{\psi}$

Proof.

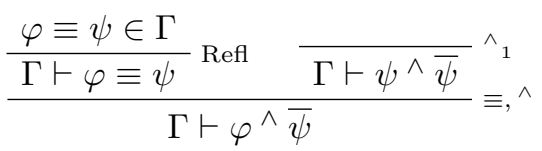

(9) $\quad \Gamma^{\prime}$, Superman $\equiv$ Clark Kent $\vdash$ Superman ${ }^{\wedge} \overline{\text { Clark Kent }}$ 2. $\Gamma^{\prime}, \varphi^{\wedge} \psi \vdash \varphi \equiv \bar{\psi}$

Proof.

$$
\begin{gathered}
\frac{\varphi^{\wedge} \psi \in \Gamma}{\Gamma \vdash \varphi^{\wedge} \psi} \text { Refl } \frac{{\overline{\Gamma \vdash \psi^{\wedge} \bar{\psi}}}^{\wedge}{ }_{1}}{\Gamma \vdash \varphi \equiv},{ }^{\wedge} \\
\Gamma^{\prime}, \text { hate }^{\wedge} \overline{\text { hate }} \vdash \text { hates } \equiv \overline{\overline{\text { hate }}}
\end{gathered}
$$

3. $\Gamma \vdash \varphi \equiv \overline{\bar{\varphi}}$

Proof.

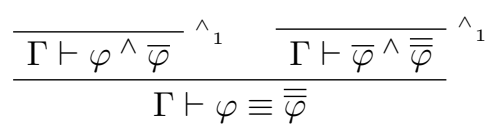

$$
\Gamma \vdash \text { human } \equiv \overline{\overline{\text { human }}}
$$

4. $\Gamma^{\prime}, \varphi \sqsubset \psi \vdash \bar{\psi} \sqsubset \bar{\varphi}$

Proof.

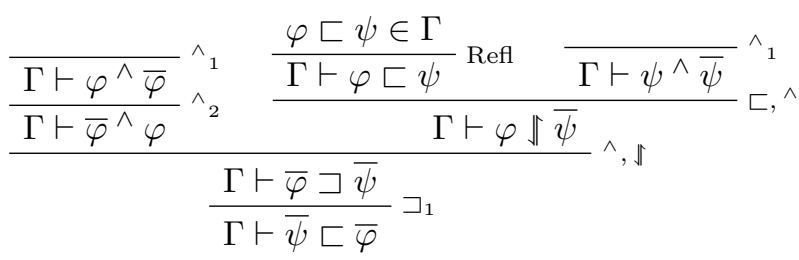

$$
\Gamma^{\prime} \text {, dane } \sqsubset \text { dog } \vdash \overline{\text { dog }} \sqsubset \overline{\text { dane }}
$$


160 / Alex J. DJalali

5. $\Gamma^{\prime}, \varphi \sqsupset \psi \vdash \bar{\psi} \sqsupset \bar{\varphi}$

Proof.

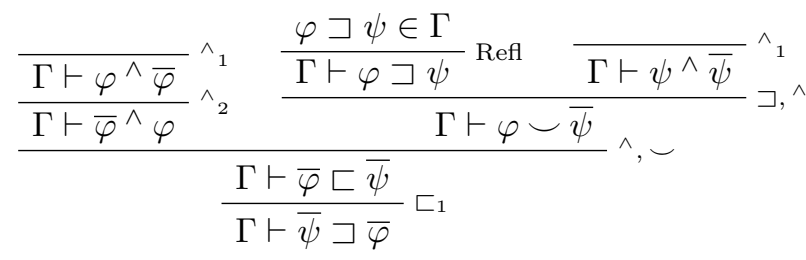

$$
\Gamma^{\prime}, \text { dog } \sqsupset \text { dane } \vdash \overline{\text { dane }} \sqsupset \overline{\text { dog }}
$$

6. $\Gamma^{\prime}, \varphi \rrbracket \psi \vdash \varphi \sqsubset \bar{\psi}$

Proof.

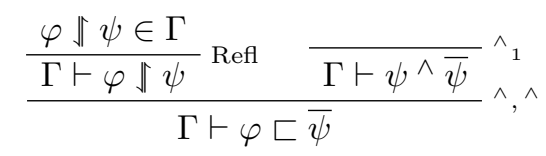

$$
\Gamma^{\prime} \text {, Dutchman } \Downarrow \text { Frenchman } \vdash \text { Dutchman } \sqsubset \overline{\text { Frenchman }}
$$

7. $\Gamma^{\prime}, \varphi \smile \psi \vdash \varphi \sqsupset \bar{\psi}$

Proof.

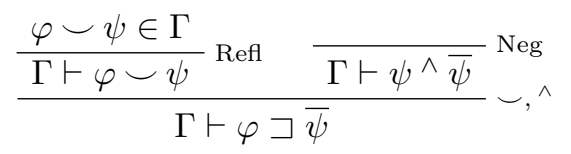

$$
\Gamma^{\prime} \text {, animal } \smile \overline{\text { human }} \vdash \text { animal } \sqsupset \overline{\overline{\text { human }}}
$$

Importantly, all of the above proofs are invertible. That is to say, $\Gamma^{\prime}, \varphi R \psi \vdash \varphi^{\prime} S \psi^{\prime}$ just in case $\Gamma^{\prime}, \varphi^{\prime} S \psi^{\prime} \vdash \varphi R \psi$.

Finally, in order to capture the notion of an inconsistent premise set, I instead add the inference rule given in definition 2.7 to the proof calculus:

Definition 2.7 (Explosion). Let $\Gamma$ be a set of synthetic terms. Then,

$$
\frac{\Gamma \vdash \varphi R \psi \quad \Gamma \vdash \varphi S \psi \quad \text { for } R \neq S}{\Gamma \vdash \varphi^{\prime} T \psi^{\prime} \text { for all } \varphi^{\prime} T \psi^{\prime}} \operatorname{Exp}
$$

is a rule of the calculus.

This rule states that if, from an arbitrary premise set $\Gamma$, two synthetic statements that claim the proper term $\varphi$ stands in a $\mathcal{M}$-relation with the proper term $\psi$ different from the other, then every synthetic term is 
derivable from that premise set. The principle of explosion has its roots in the latin term ex falso quodlibet, which means 'from a contradiction anything follows'. In this setting, Exp is the proof-theoretic realization of theorem 2.1. Now I can define what it is for a premise set to be consistent:

Definition 2.8 (Consistency). $\Gamma$ is consistent if, and only if $\Gamma \nvdash \varphi R \psi$ for some synthetic term $\varphi R \psi$.

In classical propositional logic, for example, a consistent set of premises is a set that does not prove falsum. From this definition, for any arbitrary premise set $\Gamma$ to prove every formula of a language is for that premise set to be inconsistent. So, I take what is a theorem in classical logic as my definition for inconsistency here. Theorem 2.3 gives an example of an inconsistent set:

Theorem 2.3. $\Gamma=\{\varphi \sqsubset \psi, \psi \sqsupset \vartheta, \varphi \smile \vartheta\}$ is inconsistent.

Proof.

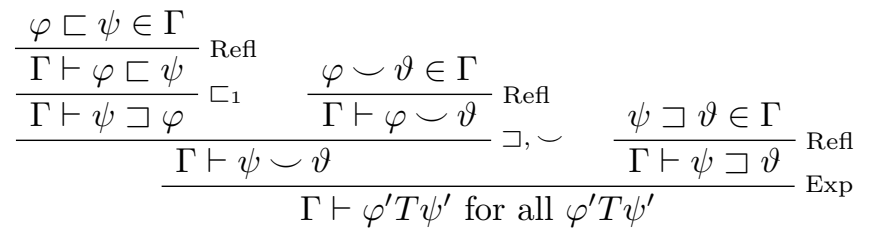

\subsection{Completeness}

Finally, the logic laid out here can be shown to be complete.

Theorem 2.4 (Completeness). Let $\Gamma$ be a set of synthetic terms. Then

$$
\Gamma \vdash \varphi R \psi \Leftrightarrow \Gamma \models \varphi R \psi
$$

As usual, soundness is trivial, but tedious, as it involves proving the statement of the theorem for all the inference rules of the calculus. I leave it as an exercise to the reader; and the remainder of this section proving the adequacy of the calculus, reasoning via the contraposition.

$$
\Gamma \nvdash \varphi R \psi \Rightarrow \Gamma \not \models \varphi R \psi
$$

The proof will proceed as normal via a model existence lemma, and essentially follows Moss (2010). The model we construct will be a term model.

Lemma 2.1 (Model Existence). Let $\Gamma$ be a set of synthetic terms. If $\Gamma$ is consistent, then $\Gamma$ has a model.

Model existence will be proved via a representation theorem.

I begin first by defining the necessary algebraic machinery. 
162 / Alex J. DJalali

Definition 2.9 (Orthoposets). An orthoposet is a tuple $(P, \leq, 0,-)$ such that

1. $(P, \leq)$ is a partial order;

2. 0 is a minimal element, i.e., $0 \leq x$ for all $x \in P$;

3. $x \leq y$ if, and only if $\bar{y} \leq \bar{x}$;

4. $\overline{\bar{x}}=x$

5. If $x \leq y$ and $x \leq \bar{y}$, then $x=0$.

An orthoposet is a partial order with a minimal element. The third clause guarantees that the contraposition holds; clause four is the law of double negation; and the final clause guarantees that any inconsistent element just is the minimal element. Importantly for me, every consistent set of premises $\Gamma$ induces an orthoposet on the domain of proper terms $\Phi$.

Lemma 2.2. If $\Gamma$ is consistent, then $\Gamma$ induces an orthoposet on $\Phi$.

Proof. For $\varphi, \psi \in \Phi$, define the relation $\leq_{\Gamma}$ as follows:

$$
\varphi \leq_{\Gamma} \psi \Leftrightarrow \Gamma \vdash \varphi \equiv \psi \text { or } \Gamma \vdash \varphi \sqsubset \psi
$$

$\leq_{\Gamma}$ induces an equivalence relation on $\Phi$ :

$$
[\varphi]_{{ }_{\Gamma}}=\left\{\psi \mid \varphi \leq_{\Gamma} \psi \text { and } \psi \leq_{\Gamma} \varphi\right\}
$$

To prove that $=_{\Gamma}$ is an equivalence relation, observe that $\varphi \leq_{\Gamma} \psi$ and $\psi \leq_{\Gamma} \varphi$ if, and only if $\Gamma \vdash \varphi \equiv \psi$ and $\Gamma \vdash \psi \equiv \varphi$, lest $\Gamma$ be inconsistent. So, the only interesting case is transitivity, which is guaranteed by the $M$-rule $\equiv, \equiv$.

Now, let $\Phi^{*}$ be the quotient $\Phi /=_{\Gamma}$ and define the relation $\leq$ as follows:

$$
[\varphi]_{=_{\Gamma}} \leq[\psi]_{=_{\Gamma}} \Leftrightarrow \forall x \in[\varphi]_{=_{\Gamma}} \forall y \in \leq[\psi]_{=_{\Gamma}}\left(x \leq_{\Gamma} y\right)
$$

Observe that $[\varphi]_{=_{\Gamma}} \leq[\psi]_{=_{\Gamma}}$ if, and only if $\forall x, y$ such that $x \in[\varphi]_{=_{\Gamma}}, y \in$ $[\psi]_{=_{\Gamma}}, \Gamma \vdash x \equiv y$ or $\forall x, y$ such that $x \in[\varphi]_{=_{\Gamma}}, y \in[\psi]_{=_{\Gamma}}, \Gamma \vdash x \sqsubset y$. Clearly, $\leq$ is a partial order. Reflexivity and anti-symmetry are trivial. Transitivity can be gotten from either $\equiv, \equiv$ or $\sqsubset, \sqsubset$.

As $\Gamma$ is assumed to be consistent, there is no $\varphi \leq \bar{\varphi}$. Therefore, add fresh elements 0,1 to $\Phi^{*}$, setting $\overline{0}=1$ and $0<x<1$ for all $x \in \Phi^{*}$. Finally, set $[\bar{\varphi}]_{=_{\Gamma}}=\overline{[\varphi}_{=_{\Gamma}}$. I claim $\left(\Phi^{*}, \leq, 0,-\right)$ is an orthposet. Condition 3 of definition 2.9 can be gotten by theorem 2.2.4, and condition 4 is obtained by theorem 2.2.3. The final condition holds vacuously, as $\Gamma$ is assumed to be consistent.

Before continuing with the proof proper, I will need a few more algebraic notions. 
Definition 2.10 (Points). A point of an orthoposet is a subset $S \subseteq P$ with the following properties:

1. If $x \in S$ and $x \leq y$, then $y \in S$ ( $S$ is upward-closed $)$;

2. For all $x$, either $x \in S$ or $\bar{x} \in S$ ( $S$ is complete), but not both ( $S$ is consistent).

A point is similar to an ultrafilter. It is closed under upward entailment and makes a decision for every element in the domain whether that element or its complement, but not both, is a member of that point.

The following lemma, due to Moss (2010), will prove useful in the construction of the necessary model.

Lemma 2.3. For a subset, $T$, of an orthoposet $P=(P, \leq, 0,-)$, the following are equivalent:

1. $T$ is a subset of a point $S$ in $P$;

2. For all $x, y \in T, x \not \leq \bar{y}$.

As is common in algebra, the notion of a morphism will play an essential role in proof by representation.

Definition 2.11 (Morphism). A morphism of orthoposets is a map $f$ such that

1. if $x \leq y$ then $f(x) \leq f(y)$;

2. $f(\bar{x})=\overline{f(x)}$;

3. $f(0)=0$.

We say that $f$ is strictly order-preserving if $x \leq y$ if, and only if $f(x) \leq f(y)$. That is to say a strict morphism is a bi-directional order and complementation preserving map.

At the outset, we stated our proof of completeness will proceed via representation. The most well-known representation theorem is Stone's, which states that every boolean algebra can be represented as a system of sets ordered by the inclusion relation. As Moss (2010) observes, it has long been established in quantum logic that every orthoposet can be represented as a system of sets also ordered by the inclusion relation (Zierler and Schlessinger 1965; Calude et al. 1999).

Theorem 2.5 (Representation). Let $P=(P, \leq, 0,-)$ be an orthoposet. There is a set, points $(P)$, and a strict morphism $f$ such that

$$
f: P \rightarrow \mathcal{P}(\operatorname{points}(P))
$$

The proof is gotten by defining $f(x)=\{S \in \operatorname{point}(P) \mid x \in S\}$. I refer the reader to Moss (2010) for the remainder of the proof. 
We are now equipped with all the algebraic machinery we need to prove lemma 2.1 .

Proof. As $\Gamma$ is consistent, I invoke lemma 2.2 and theorem 2.5 to conclude $\Phi$ can be represented as a system of sets. In particular, define $g: \Phi \rightarrow \Phi_{=_{\Gamma}}$ such that $\varphi \mapsto[\varphi]_{=_{\Gamma}}$ and let $f$ be the strict morphism defined in theorem 2.5.

Now, let $\mathbb{M}=\left(\operatorname{points}\left(\Phi^{*}\right), A A A \cdot B B B\right)$, such that $A A A \cdot B B B=$ $g \circ f$, the composition of $g$ with $f$. I claim that $\mathbb{M}$ a synthetic model. I must check that it has the properties stipulated in definition 2.2. The first condition on the valuation function can be shown to hold as follows:

$$
\begin{aligned}
A A A \bar{\varphi} B B B & =f(g(\bar{\varphi})) & & \text { by construction of } \\
& =f(\overline{g(\varphi)}) & & A A A \cdot B B B \\
& =\overline{f(g(\varphi))} & & {[\bar{\varphi}] } \\
& =\operatorname{points}\left(\Phi^{*}\right)-f(g(\varphi)) & & \text { by is a morphism } \\
& =\operatorname{points}\left(\Phi^{*}\right)-A A A \varphi B B B & & \text { by construction of } \\
& & & A A A \cdot B B B
\end{aligned}
$$

To show that no proper term is interpreted vacuously two conditions must be shown to hold. I begin by proving no proper term is interpreted as $\varnothing$. Consider the set $T=\left\{[\varphi]_{=_{\Gamma}}\right\} .[\varphi]_{=_{\Gamma}} \not{Z}[\bar{\varphi}]_{=_{\Gamma}}$, else $\Gamma$ would be inconsistent, contradicting the initial assumption. So, $[\varphi]_{=_{\Gamma}} \not{[} \overline{[\varphi]}_{=_{\Gamma}}$, and applying lemma 2.3, I conclude $T$ to be a subset of some point $S \in \operatorname{points}\left(\Phi_{\Gamma}^{*}\right)$. By the construction of $A A A \cdot B B B, T \in A A A \varphi B B B=$ $f(g(\varphi))$, a non-empty set.

To show that no proper term is interpreted as points $\left(\Phi^{*}\right)$, observe that, by the above, $A A A \bar{\varphi} B B B$ is non-empty. Let $S$ be such a witness. Suppose that $S \in A A A \varphi B B B$. So, $g(\varphi) \in S$, but so is $g(\bar{\varphi})=\overline{g(\varphi)}$ by the construction of the function. This contradicts the fact that $S$ is consistent. So, $S \notin A A A \varphi B B B$ and we have found the necessary point.

Lemma 2.4. Let $\mathbb{M}$ be the model constructed above. Then

$$
\mathbb{M} \models \varphi R \psi \Leftrightarrow \Gamma \vdash \varphi R \psi
$$

Proof. This proof relies on the fact that both $g$ and $f$ are monotone functions. The most important cases, here, are where $R=\equiv$ and $R=\sqsubset$, as every other case can be derived from these two, as theorem 2.2 indicates. Technically, one need consider the various possibilities where $\varphi=\psi$ or $\psi=\bar{\varphi}$, etc. However, most of these cases contradict the fact 
that $\Gamma$ was assumed to be consistent, and the remainder are analagous to the following.

1. $R=\equiv$

$$
\begin{aligned}
\Gamma \vdash \varphi \equiv \psi & \Leftrightarrow \varphi \leq_{\Gamma} \psi \text { and } \psi \leq_{\Gamma} \varphi \\
& \Leftrightarrow g(\varphi)=g(\psi) \\
& \Leftrightarrow f(g(\varphi))=f(g(\psi)) \\
& \Leftrightarrow A A A \varphi B B B=A A A \psi B B B \\
& \Leftrightarrow \Gamma \models \varphi \equiv \psi
\end{aligned}
$$

2. $R=\sqsubset$

$$
\begin{aligned}
\Gamma \vdash \varphi \sqsubset \psi & \Leftrightarrow \varphi \leq_{\Gamma} \psi \text { and } \psi \not_{\Gamma} \varphi \\
& \Leftrightarrow g(\varphi) \leq g(\psi) \text { and } g(\psi) \not \leq g(\varphi) \\
& \Leftrightarrow f(g(\varphi)) \subset f(g(\psi)) \\
& \Leftrightarrow A A A \varphi B B B \subset A A A \psi B B B \\
& \Leftrightarrow \Gamma \models \varphi \sqsubset \psi
\end{aligned}
$$

The remainder of the proof of theorem 2.4 is gotten in the standard way.

\section{Conclusion}

When I first began work in this area, my intention was to understand the underlying logic of M\&M's join table. I did so by giving it a natural set-theoretic semantics and a simple proof calculus. Having done so, I was able to prove completeness via representation. I think, though, that there are broader implications in this line of research.

First and foremost, the logic I have presented here can be understood as a logic underlying a NL lexicon like that of English, if the MacCartney relations are interpreted as lexical semantic relations. More specifically, a synthetic logic can be understood as being the logic of a lexical network like that of, say, WordNet, as it allows us to (begin to) answer the question: If an expression $\alpha$ stands in a lexical semantic relation with $\beta$, and $\beta$ stands in a (possibly different) lexical semantic relation with $\gamma$, what lexical semantic relation does $\alpha$ stand in with $\gamma$ ?

Second, I conjecture that the logic here can be embedded in Moss's (2010) syllogistic fragment that contains complements, suggesting that there is much work left to be done with synthetic logic itself. Third, I have not begun to explore the complexity of a synthetic language. If we are interested in logics that have (viable) computational reflexes, to 
determine complexity results of a synthetic logic would be an obvious next step in this line of research.

\section{References}

Calude, Cristian, Peter Hertling, and Karl Svozil. 1999. Embedding quantum universes in classical ones. Foundations of Physics 29:349-379. 10.1023/A:1018862730956.

Dowty, David. 1994. The role of negative polarity and concord marking in natural language reasoning. In M. Harvey and L. Santelmann, eds., Proceedings of Semantics and Linguistic Theory 4, pages 114-144. CLC Publications.

Gilad, Ben Avi and Nissim Francez. 2005. Proof-theoretic semantics for a syllogistic fragment. In Proceedings of the Fifteenth Amsterdam Colloquium, pages 9-15. ILLC/Department of Philosophy, University of Amsterdam.

MacCartney, Bill. 2009. Natural Language Inference. Ph.D. thesis, Stanford University.

MacCartney, Bill and Christopher Manning. 2009. An extended model of natural logic. In The Eighth International Conference on Computational Semantics (IWCS-8). Tilburg, The Netherlands.

Moss, Larry. 2008. Completeness theorems for syllogistic fragments. In F. Hamm and S. Kepser, eds., Logics for Linguistic Structures, pages 143173. Mouton de Gruyter. Draft.

Moss, Larry. 2010. Syllogistic logic with complements. In J. van Benthem, A. Gupta, and E. Pacuit, eds., Games, Norms and Reasons: Logic at the Crossroads, pages 185-203. Springer Synthese Library Series.

Moss, Larry. 2012. The soundness of internalized polarity marking. Studia Logica 100:683-704.

Moss, Lawrence S. 2009. Natural logic and semantics. In M. Aloni, H. Bastiaanse, T. de Jager, P. van Ormondt, and K. Schulz, eds., Preproceedings of the 17th Amsterdam Colloquium, pages 71-80. University of Amsterdam.

Popper, Karl. 1968. The Logic of Scientific Discovery. Harper Torchebooks.

Russell, Bertrand. 1905. On denoting. Mind 14(56):479-493. ArticleType: research-article / Full publication date: Oct., 1905 / Copyright (C) 1905 Oxford University Press.

Valencia, Sanchez. 1991. Studies on Natural Logic and Categorial Grammar. Ph.D. thesis, Universiteit van Amsterdam.

van Benthem, Johan. 1986. Essays in Logical Semantics. Dodrecht: D. Reidel. van Benthem, Johan. 2008. A brief history of natural logic. In M. Chakraborty, B. Löwe, M. Nath Mitra, and S. Sarukki, eds., Logic, Navya-Nyaya and Applications: Homage to Bimal Matilal.

Zierler, Neal and Michael Schlessinger. 1965. Boolean embeddings of orthomodular sets and quantum and logic. Duke Math Journal 32(2). 